\title{
First Genomic Resource of the Columbia Lance Nematode Hoplolaimus columbus
}

\author{
Xinyuan Ma, ${ }^{1, \dagger}$ J. Antonio Baeza, ${ }^{2,3,4}$ Vincent P. Richards, ${ }^{2}$ and Paula Agudelo ${ }^{1}$ \\ ${ }^{1}$ Department of Plant and Environmental Sciences, Clemson University, Clemson, SC 29634, U.S.A. \\ ${ }^{2}$ Department of Biological Sciences, 132 Long Hall, Clemson University, Clemson, SC 29634, U.S.A. \\ ${ }^{3}$ Smithsonian Marine Station at Fort Pierce, 701 Seaway Drive, Fort Pierce, FL 34949, U.S.A. \\ ${ }^{4}$ Departamento de Biología Marina, Facultad de Ciencias del Mar, Universidad Católica del Norte, \\ Larrondo 1281, Coquimbo, Chile
}

\begin{abstract}
The Columbia lance nematode Hoplolaimus columbus has been reported frequently from North America due to its negative impact on agricultural production. In this study, for the first time, we sequenced the whole genome of a female specimen by using whole-genome amplification and Illumina MiSeq. Data were de novo assembled to form scaffolds of $205.75 \mathrm{Mbp}$ consisting of 118,374 contigs. The largest scaffold was $636,881 \mathrm{bp}$. Benchmarking Universal Single-Copy Orthologs completeness was $66.6 \%$ (eukaryotic dataset), and $>8,000$ unique genes were predicted by GeneMark-ES. In total, 61,855 protein sequences were predicted by AUGUSTUS, and 10,085 of them were annotated by PANNZER2 with at least one function. These data will provide valuable resources for studies focusing on pathogenicity and phylogenomics of plant-parasitic nematodes.
\end{abstract}

\section{Genomic Resource Announcement}

Lance nematodes (Hoplolaimus spp.) is a genus of soil-inhabiting plant parasites distributed around the world that includes $>30$ species (Ma et al. 2011, 2019; Sher 1963). Hoplolaimus columbus, also known as the Columbia lance nematode, was first reported in 1963 in Columbia, SC, and later was found in North Carolina, Georgia, Alabama, and Louisiana (Astudillo and Birchfield 1980; Gazaway and Armstrong 1994; Holguin et al. 2015, 2016; Koenning et al. 1999; Krusberg and Sasser 1956). Most of its close relatives are reported in Pakistan, India, China, and Japan (Siddiqi 2000). These lance nematodes have a distinct cephalic region and a welldeveloped stylet (Sher 1963) that enable them to be migratory ecto- and endoparasites. They can destroy cortex cells, which results in the extension of necrotic lesions (Krusberg and Sasser 1956; Lewis and Fassuliotis 1982). As a significant threat to agricultural plants in the southeastern United States, $H$. columbus can cause up to $25 \%$ production losses for cotton, and its damage on soybean production can be as high as 70\% (Appel and Lewis 1984; Lewis et al. 1976; Mueller and Sanders 1987; Noe 1993). In spite of its importance to phytopathology, no genomic data are available yet. In this study, for the first time, we provide a genomic resource for an $\mathrm{H}$. columbus female isolate from North America. In addition, a complete mitochondrial chromosome of H. columbus was de novo assembled using NOVOPlasty (Dierckxsens et al. 2017) and has been reported (Ma et al. 2020).

In April 2017, H. columbus specimens were collected from the Edisto Research Center in Blackville, SC. Specimens were extracted from soil samples (Jenkins 1964), then diagnosed and identified according to results of morphological observation and molecular methods (Bae et al. 2009; Handoo and Golden 1992; Ma et al. 2011). This species identification was later validated by aligning the assembled mitochondrial genome of the isolate with COX1 sequences of H. columbus in GenBank (Ma et al. 2020). The whole genomic DNA was first extracted from a

${ }^{\dagger}$ Corresponding author: X. Ma; xm@g.clemson.edu

The author(s) declare no conflict of interest.

Accepted for publication 17 March 2021.
Funding

Support was provided by the South Carolina Cotton Board project SC15-130.

Keywords

de novo assembly, genome, Hoplolaimus columbus, lance nematode, MiSeq 
Table 1. Genome assembly information of Hoplolaimus columbus analyzed using QUAST ${ }^{\mathrm{a}}$

\begin{tabular}{|c|c|c|}
\hline \multirow[b]{2}{*}{ Sample information } & \multicolumn{2}{|c|}{ Hoplolaimus columbus } \\
\hline & Contigs & Scaffolds \\
\hline \multicolumn{3}{|l|}{ Assembly metrics } \\
\hline Number of contigs & 118,374 & 117,809 \\
\hline Number of contigs $(\geq 1,000 \mathrm{bp})$ & 67,667 & 67,120 \\
\hline Number of contigs ( $\geq 5,000 \mathrm{bp})$ & 5,653 & 5,759 \\
\hline Number of contigs $(\geq 1,0000 \mathrm{bp})$ & 862 & 923 \\
\hline Number of contigs ( $\geq 25,000 \mathrm{bp})$ & 59 & 51 \\
\hline Number of contigs ( $\geq 50,000 \mathrm{bp})$ & 40 & 32 \\
\hline Largest sequence & 386,658 & 636,881 \\
\hline Total length & $205,719,153$ & $205,753,054$ \\
\hline $\mathrm{N}_{50}$ & 2,321 & 2,341 \\
\hline $\mathrm{N}_{75}$ & 1,198 & 1,199 \\
\hline $\mathrm{L}_{50}$ & 22,274 & 21,829 \\
\hline $\mathrm{L}_{75}$ & 53,864 & 53,302 \\
\hline GC (\%) & 39.42 & 39.42 \\
\hline \multicolumn{3}{|l|}{ Mismatches } \\
\hline Number of Ns & 0 & 29,168 \\
\hline Number of Ns per 100 kbp & 0 & 14.18 \\
\hline \multicolumn{3}{|l|}{ Predicted genes (GeneMark-ES) } \\
\hline Number of predicted genes (unique) & - & 8,242 \\
\hline Number of predicted genes ( $\geq 0 \mathrm{bp}$ ) & - & $15,691+0$ part \\
\hline Number of predicted genes ( $\geq 300 \mathrm{bp})$ & - & $9,303+0$ part \\
\hline Number of predicted genes $(\geq 1,500 \mathrm{bp})$ & - & 714 + 0 part \\
\hline Number of predicted genes $(\geq 3,000 \mathrm{bp})$ & - & $76+0$ part \\
\hline
\end{tabular}

single nematode (female) using a Sigma Extract-N-Amp kit (XNAT2) (Sigma-Aldrich) with an optimized extraction protocol (Ma et al. 2011), then was amplified to obtain an adequate quantity using an Illustra Ready-To-Go GenomiPhi V3 DNA Amplification kit (GE Healthcare). Three replications of whole genomic DNA amplification were performed, DNA concentration in each step was checked using a Qubit fluorometer (Invitrogen), and the one with the highest DNA concentration was selected for library preparation using the Nextera XT kit (Illumina). The library quality was checked using a Bioanalyzer 2100 (Agilent Technologies), then sequenced on the Illumina MiSeq platform using the $v 3$ kit chemistry.

The sequencing generated 40,837,196 paired-end (PE) 300-bp reads for $H$. columbus. Raw reads were deposited into the NCBI Sequence Read Archive (SRA) with the accession number SRR12516078. The NCBI-SRA taxonomy analysis identified $49.79 \%$ of raw reads as bacteria, which may be potential contamination from nematode intestine. Raw reads were trimmed using Trimmomatic 0.36 (Bolger et al. 2014) to clean adapters. Phred score threshold was 30 . The quality of trimmed reads was checked using FastQC (https://www.bioinformatics.babraham.ac.uk/ projects/fastqc/), and synchronized using Fastq-pair (Edwards and Edwards 2019). Those trimmed high-quality PE reads then were de novo assembled using SPAdes (3.14.1) (Nurk et al. 2013). Abyss-Sealer (Paulino et al. 2015) was used for gap-closing and scaffolding. The assembly was analyzed on the QUAST webserver (http://cab.cc.spbu.ru/quast) (Gurevich et al. 2013) (Table 1).

The total size of assembled scaffolds was $205.75 \mathrm{Mbp}$. The longest scaffold was 636,881 bp, $\mathrm{N}_{50}$ was 2,341 bp, and GC content was 39.42\%. GeneMark-ES (Lomsadze et al. 2005) on the QUAST webserver predicted 8,242 unique genes. The Benchmarking Universal Single-Copy Orthologs (BUSCO 3.02) analysis (Seppey et al. 2019) was performed on the webserver GenomeQC (https://genomeqc.maizegdb.org/) (Manchanda et al. 2020) with the eukaryote_odb9 dataset. The BUSCO results found $66.6 \%$ of BUSCO genes in the assembly, including $41.2 \%$ complete genes (38.6\% single copy $+2.6 \%$ duplicated copy) and $25.4 \%$ fragmented genes. AUGUSTUS analysis (Stanke and Morgenstern 2005) on WebAUGUSTUS Service (http://bioinf. uni-greifswald.de/webaugustus/) predicted 61,855 protein sequences referring to the database of Caenorhabditis. The protein sequences were then analyzed on PANNZER2 (http://ekhidna2. biocenter.helsinki.fi/sanspanz/) (Törönen et al. 2018) for functional annotation and gene ontology analysis, resulting in 10,058 protein sequences annotated with at least one function. The assembly scaffolds >2,000 bp have been processed and filtered by NCBI and deposited into GenBank. 


\section{Data Deposition}

Raw reads from the MiSeq platform have been deposited in the NCBI Sequence Read Archive with the accession number PRJNA659263. The accession number of deposited assembled scaffolds is JAEHOM000000000 in GenBank. The mitochondrial chromosome sequence has been deposited in NCBI GenBank with accession number MH657221.

\section{Literature Cited}

Appel, J. A., and Lewis, S. A. 1984. Pathogenicity and reproduction of Hoplolaimus columbus and Meloidogyne incognita on Davis soybean. J. Nematol. 16: 349-355.

Astudillo, G. E., and Birchfield, W. 1980. Pathology of Hoplolaimus columbus on sugarcane. Phytopathology 70:565.

Bae, C.-H., Robbins, R. T., and Szalanski, A. L. 2009. Molecular identification of some Hoplolaimus species from the USA based on duplex PCR, multiplex PCR and PCR-RFLP analysis. Nematology 11:471-480.

Bolger, A. M., Lohse, M., and Usadel, B. 2014. Trimmomatic: A flexible trimmer for Illumina sequence data. Bioinformatics 30:2114-2120.

Dierckxsens, N., Mardulyn, P., and Smits, G. 2017. NOVOPlasty: De novo assembly of organelle genomes from whole genome data. Nucleic Acids Res. 45:e18.

Edwards, J. A., and Edwards, R. A. 2019. Fastq-pair: Efficient synchronization of paired-end fastq files. BioRxiv.

Gazaway, W. S., and Armstrong, B. A. 1994. First report of Columbia lance nematode (Hoplolaimus columbus) on Cotton in Alabama. Plant Dis. 78:640.

Gurevich, A., Saveliev, V., Vyahhi, N., and Tesler, G. 2013. QUAST: Quality assessment tool for genome assemblies. Bioinformatics 29:1072-1075.

Handoo, Z., and Golden, A. M. 1992. A key and diagnostic compendium to the species of the genus Hoplolaimus Daday, 1905 (Nematoda: Hoplolaimidae). J. Nematol. 24:45-53.

Holguin, C. M., Baeza, J. A., Mueller, J. D., and Agudelo, P. 2015. High genetic diversity and geographic subdivision of three lance nematode species (Hoplolaimus spp.) in the United States. Ecol. Evol. 5:2929-2944.

Holguin, C. M., Ma, X., Mueller, J. D., and Agudelo, P. 2016. Distribution of Hoplolaimus species in soybean fields in South Carolina and North Carolina. Plant Dis. 100: 149-153.

Jenkins, W. R. 1964. A rapid centrifugal-flotation technique for separating nematodes from soil. Plant Dis. 48:692.

Koenning, S. R., Overstreet, C., Noling, J. W., Donal, P. A., Becker, J. O., and Fortnum, B. A. 1999. Survey of crop losses in response to phytoparasitic nematodes in the United States for 1994. J. Nematol. 31:587-618.

Krusberg, L. R., and Sasser, J. N. 1956. Host-parasitic relationship of the lance nematode on cotton roots. Phytopathology 46:505-510.

Lewis, S. A., and Fassuliotis, G. 1982. Lance nematodes, Hoplolaimus spp., in the Southern United States. Pages 127-138 in: Nematology in the Southern Region of the United States. R. D. Riggs, ed. Southern Cooperative Series Bulletin, Issue 276. University of Illinois at Urbana-Champaign, Urbana, IL, U.S.A.

Lewis, S. A., Smith, F. H., and Powell, W. M. 1976. Host-parasite relationships of Hoplolaimus columbus on cotton and soybean. J. Nematol. 8:141-145.
Lomsadze, A., Ter-Hovhannisyan, V., Chernoff, Y. O., and Borodovsky, M. 2005. Gene identification in novel eukaryotic genomes by self-training algorithm. Nucleic Acids Res. 33:6494-6506.

Ma, X., Agudelo, P., Mueller, J. D., and Knap, H. T. 2011. Molecular characterization and phylogenetic analysis of Hoplolaimus stephanus. J. Nematol. 43:25-34.

Ma, X., Agudelo, P., Richards, V. P., and Baeza, J. A. 2020. The complete mitochondrial genome of the Columbia lance nematode, Hoplolaimus columbus, a major agricultural pathogen in North America. Parasit. Vectors 13:321.

Ma, X., Robbins, R. T., Bernard, E. C., Holguín, C. M., and Agudelo, P. 2019. Morphological and molecular characterisation of Hoplolaimus smokyensis $\mathrm{n}$. $\mathrm{sp}$. (Nematoda: Hoplolaimidae), a lance nematode from Great Smoky Mountains National Park, USA. Nematology 21:923-935.

Manchanda, N., Portwood, J. L., Woodhouse, M. R., Seetharam, A. S., LawrenceDill, C. J., Andorf, C. M., and Hufford, M. B. 2020. GenomeQC: A quality assessment tool for genome assemblies and gene structure annotations. BMC Genomics 21:193.

Mueller, J. D., and Sanders, G. B. 1987. Control of Hoplolaimus columbus on late-planted soybean with aldicarb. J. Nematol. 19:123-126.

Noe, J. P. 1993. Damage functions and population changes of Hoplolaimus columbus on cotton and soybean. J. Nematol. 25:440-445.

Nurk, S., Bankevich, A., Antipov, D., Gurevich, A., Korobeynikov, A., Lapidus, A., Prjibelsky, A., Pyshkin, A., Sirotkin, A., Sirotkin, Y., and Stepanauskas, R. 2013. Assembling genomes and mini-metagenomes from highly chimeric reads. Pages 158-170 in: Annu. Int. Conf. Res. Comput. Mol. Biol. Springer, Berlin, Heidelberg.

Paulino, D., Warren, R. L., Vandervalk, B. P., Raymond, A., Jackman, S. D., and Birol, I. 2015. Sealer: A scalable gap-closing application for finishing draft genomes. BMC Bioinf. 16:230.

Seppey, M., Manni, M., and Zdobnov, E. M. 2019. BUSCO: Assessing genome assembly and annotation completeness. Pages 227-245 in: Gene Prediction. M. Kollmar, ed. Methods in Molecular Biology, vol. 1962. Humana, New York, NY, U.S.A.

Sher, S. A. 1963. Revision of the Hoplolaiminae (Nematoda) li. Hoplolaimus Daday, 1905 and Aorolaimus N. Gen. Nematologica 9:267-295.

Siddiqi, M. R. 2000. Tylenchida Parasites of Plants and Insects, 2nd ed. CABI Publishing, Wallingford, U.K.

Stanke, M., and Morgenstern, B. 2005. AUGUSTUS: A web server for gene prediction in eukaryotes that allows user-defined constraints. Nucleic Acids Res. 33: W465-W467.

Törönen, P., Medlar, A., and Holm, L. 2018. PANNZER2: A rapid functional annotation web server. Nucleic Acids Res. 46:W84-W88. 